\title{
La innovación en la micro y la pequeña empresa (MYPE): no solo factible, sino accesible ${ }^{1}$
}

\author{
Fátima Ponce Regalado \\ Pontificia Universidad Católica del Perú \\ fponce@pucp.edu.pe \\ Emilio Zevallos Vallejos \\ Facultad Latinoamericana de Ciencias Sociales (FLACSO), Sede Costa Rica \\ ezevallosv535@ulacit.ed.cr
}

\footnotetext{
1. Este artículo está basado en la ponencia presentada, como parte de la sección Call for Papers, durante el I Congreso Internacional de Ciencias de la Gestión: Gestión de la Innovación e Innovación en la Gestión, desarrollado del 29 de setiembre al 2 de octubre de 2015 en la Pontificia Universidad Católica del Perú por el Departamento Académico de Ciencias de la Gestión y la Facultad de Gestión y Alta Dirección.
} 
El presente artículo busca señalar que la innovación en las MYPES latinoamericanas no solo es necesaria, sino accesible, y que no se da como tradicionalmente se entiende a la innovación (asociada a la tecnología). La hipótesis es que innovar en la MYPE no es imposible ni necesariamente costoso; por el contrario, es necesario y accesible, pero diferente debido a que su innovación no es tanto tecnológica sino más centrada en procesos o modelos de gestión. De hecho, ya varias de estas empresas la están implementando para lograr impulsar su crecimiento y sostenibilidad.

Se evidencia esta hipótesis y se muestra que en la región la innovación en las MYPES se presenta en sus procesos y/o modelos de gestión - aspectos donde las MYPES tienen grandes oportunidades de innovar y mejorar su productividad-, como se observa del análisis de las estadísticas sobre el tema tanto de Costa Rica como de Perú. Se busca caracterizar a las MYPES, su liderazgo y el entorno de innovación en el que se desenvuelven a fin de hacer visibles las competencias requeridas y el tipo de innovación que desarrollan (más en procesos y modelos de gestión y menos en productos o en tecnología). Asimismo, se presentan dos casos exitosos en generación de innovaciones con el propósito de ver cómo las MYPES son capaces de aplicar medidas novedosas y cambiar el rumbo cuando es necesario.

Palabras clave: innovación, tecnología, procesos, modelos de gestión, MYPE

The present paper seeks to point out that innovation is also accessible and necessary for the micro and small entreprises (MSE) in Latin America. But it is not given as traditionally understood as associated to technology. The hypothesis is that innovate in the MSE is not impossible nor necessarily expensive; on the contrary it is necessary and feasible, but different because it is related less to techonology than to processes or management models. In fact, several of these small businesses are making that in order to promote their growth and sustainability.

To evidence this hypothesis and show that the regional innovation is rather in processes and/or management models, aspects where small firms have great opportunities to innovate and improve their productivity, this work analyzes statistics both in Costa Rica and Peru. The purpose is to define the MSEs main features, the owners skills and their innovation environment, in order to make visible its competences and the kind of innovation focus more in processes and management models and less in products or technology. It also presents two successful cases in generation of innovations with the purpose of presenting how the MSE are able to apply new measures and redirect their course when necessary.

Keywords: innovation, technology, process, management models, micro and small business 


\section{Antecedentes}

Las micro y pequeñas empresas (MYPES) son un conjunto heterogéneo de unidades económicas (en términos de tamaño, sector, región, tipo de producto/servicio desarrollado, etcétera), que ha aumentado sostenidamente en las últimas décadas en América Latina. Las MYPES constituyen un creciente sector en la economía, llegando a representar en algunos países hasta el $90 \%$ de todas las unidades económicas. Tienen gran potencial para los países en desarrollo, fundamentalmente por su contribución al PBI, la generación de empleo y la reducción de las desigualdades regionales; así como por su potencial de desarrollo innovador (Zevallos, 2003). Berry señala que: "El crecimiento e incremento de productividad del sector mipyme [micro, pequeña y mediana empresa] no solo es pertinente, sino crucial, en la creación de empleo y desarrollo de la región» $(2002$, p. 5). Sin embargo, una adecuada medición de su presencia en la economía es una tarea pendiente de las instituciones públicas debido especialmente a su dispersión geográfica, a la dificultad de identificarlas debido a su tamaño y al problema de informalidad que aún existe alrededor de muchos de estos agentes económicos².

Por su importancia, estos actores empresariales deberían ocupar un papel destacado en las políticas públicas de los países de la región; sin embargo, con frecuencia son poco considerados en los momentos críticos de diseño/implementación de las políticas, especialmente las de fomento. Al mismo tiempo, ellos mismos no son conscientes de la fortaleza y capacidad de negociación que les da su número y alta dispersión territorial.

Adicionalmente, es posible señalar que los propios empresarios de estas unidades económicas son heterogéneos en términos de educación —-formal e informal—, experiencia laboral y empresarial, nivel de contactos, grado de involucramiento del empresario en el negocio, solvencia financiera, etcétera; por lo que las acciones para fortalecerlas requieren de un adecuado entendimiento no solo desde las anteriores perspectivas, sino también sobre cómo es la senda de aprendizaje de las empresas y de quienes las dirigen.

\section{Planteamiento del problema}

La evidencia internacional (Zevallos, 2003; Obando, Rojas \& Zevallos, 2008) muestra que las empresas de menor tamaño (micro y pequeña) por lo general no adoptan innovaciones, o bien si lo hacen no son plenamente conscientes de ello. Existe alrededor de esta escasez de innovación la percepción de que innovar es costoso, requiere de conocimientos técnicos avanzados, etcétera, aunque también se observa que existe poco acceso a información sobre innovaciones y, de alguna forma, estas empresas viven en un entorno reacio al cambio.

2. Al respecto, para el caso del Perú, Villarán y Mifflin señalan que: «Este argumento es mucho más relevante si se trata de microempresas, cuya mayoría está en la informalidad [...] Otro problema para medir la presencia de estos sectores empresariales ha sido que las estadísticas de algunas entidades públicas fueron desactivadas, como parte del esfuerzo de simplificación de trámites para las unidades económicas de pequeña dimensión. Este es el caso del Ministerio de la Producción (PRODUCE) que dejó de pedir información a través del Registro de Productos Industriales (RPIN)» (2009, p. 8). En el caso de Costa Rica, siendo el país de Centroamérica que cuenta con los mejores indicadores económicos, no tiene un censo de empresas, por lo que la forma de aproximar el número de empresas es por el registro a la seguridad social (indicador clave de formalidad), o bien desde la encuesta de hogares (que más bien aproxima a la informalidad). 
Lo anterior parte de la creencia generalizada de que la innovación se encuentra, fundamentalmente, referida a la tecnología y que ella se adopta en el producto. Sin embargo, no se considera que el grueso de la innovación en la región latinoamericana no es tecnológica, sino más bien en procesos y/o modelos de gestión (Obando, Rojas \& Zevallos, 2008). Esos son ámbitos centrales del negocio en donde la MYPE tiene grandes oportunidades de innovar y mejorar su productividad.

De ahí la importancia de visibilizar que la innovación también es accesible a las empresas de menor tamaño, razón por la cual postulamos en este artículo que innovar en la MYPE no es imposible ni necesariamente costoso, sino más bien accesible y necesario, pero diferente.

\section{Objetivo}

El objetivo del presente artículo es presentar evidencia de que es posible generar innovaciones sin necesariamente invertir fuertes sumas de dinero o desarrollar tecnología sofisticada, y que sus resultados permiten aumentar la productividad y competitividad. Sin embargo, para ello será necesario desarrollar competencias, cambiar hábitos y redefinir estrategias, entre otras acciones relacionadas a la cultura y gestión de la innovación en estas organizaciones.

\section{Revisión de literatura}

\subsection{Heterogeneidad}

Un elemento que caracteriza a las MYPES y al empresario que las dirige es su heterogeneidad. Viendo a la empresa y al empresario de esa forma, el proceso de desarrollo empresarial también podría ser considerado como heterogéneo. Sin embargo, y a pesar de esa consideración, podemos considerarlo semejante al proceso de crecimiento de los individuos (nacimiento, infancia, niñez, adolescencia, adultez), el cual atraviesa por ciertos hitos (Zevallos, 2012). De esta forma, el empresario atraviesa por determinadas etapas que marcan su crecimiento y que son relativamente comunes a todas las empresas.

Al respecto, el Global Entrepreneurship Monitor o GEM (Amorós, Guerra \& Carrillo, 2008) desarrolló un modelo para describir este proceso, el cual considera tres grandes etapas en el desarrollo de la empresa:

a. Una de concepción, donde lo que se resalta es el paso de la idea de negocio a la realización del mismo.

b. El nacimiento, que se orienta a la etapa en que la empresa surge y llega hasta sus primeros tres y medio años, que son momentos críticos para ver si es capaz de sobrevivir o no.

c. La persistencia, cuando la empresa ha superado los 42 meses, tiempo considerado por el GEM como suficiente para saber si la empresa es viable en el mediano-largo plazo (el gráfico 1 muestra el proceso). 
A la vez, encontramos a la mayor parte de las MYPES — particularmente las microempresas - en situación de subsistencia y con limitadas opciones de crecimiento. Investigaciones en esa línea (OECD, 1997; Zevallos, 2001) señalan que solo una pequeña parte de ellas (cercana al $25 \%$ ) será capaz de crecer y convertirse en una unidad económica con posibilidades de desarrollo; es decir, el $75 \%$ de todas las microempresas están condenadas a la subsistencia. Partiendo de esa estadística y reconociendo la importancia de las MYPES en la economía, nos preguntamos: ¿qué podemos hacer para promover el desarrollo de este importante y heterogéneo segmento empresarial y generar las condiciones para su crecimiento y sostenibilidad?

\section{Gráfico 1: Modelo del GEM}

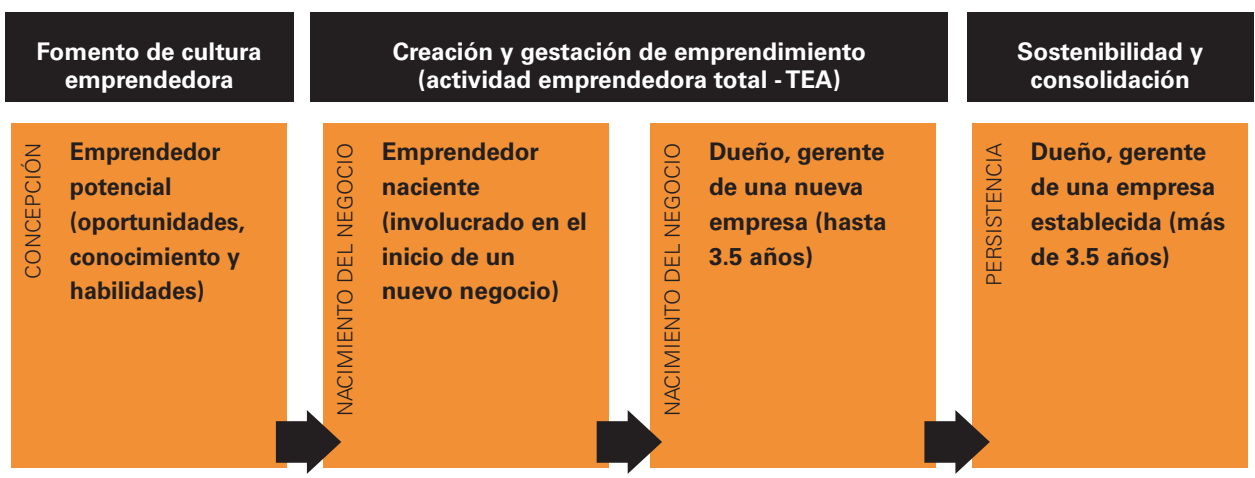

Fuente: elaboración propia sobre la base de Amorós, Guerra y Carrillo (2008).

\subsection{Innovación y competencias}

Existen al menos dos ámbitos en los que es posible generar acciones de política pública para fortalecer el potencial de desarrollo de las empresas de menor tamaño:

a. En primer lugar, es claro que «innovación» es la palabra clave para sacar a las empresas de su estado de estancamiento y llevarlas a nuevos niveles de crecimiento.

b. En segundo lugar, la visión de los gobiernos, agencias de cooperación e incluso los propios proveedores de servicios de desarrollo empresarial están orientándose hacia un cambio en la forma de entender los procesos de enseñanza-aprendizaje, más aún en un entorno digital. En ese sentido, el enfoque por competencias ${ }^{3}$ se hace cada vez más importante en el desarrollo de capacidades empresariales, razón por la cual su estudio se convierte en una necesidad creciente.

3. Se entiende por "competencias» "[...] las aptitudes, conocimientos y destrezas necesarias para cumplir exitosamente las actividades que componen una función laboral, según estándares definidos por el sector productivo". Esta definición fue tomada de la Ley N²0.267 (Sistema Nacional de Certificación de Competencias Laborales de Chile). 
Entonces, de un lado tenemos a la innovación, entendida desde una idea nueva que tiene valor para el público, sea bajo el formato de una mejora en un producto existente o bien la introducción de uno nuevo. Ella puede darse en el producto o en su proceso productivo; además, puede ser tecnológica, organizacional o en el modelo de negocio ${ }^{4}$ y también incremental o radical. El reto del emprendedor frente a la innovación es mejorar la calidad y productividad, y con ello los ingresos de su negocio y la sostenibilidad de su emprendimiento. Sin embargo, la clave en la innovación es que pueda pasar la «prueba del mercado»; es decir, que tenga demanda.

En un estudio realizado para Centroamérica hace algunos años (Obando, Rojas \& Zevallos, 2008) se señala que el grueso de las innovaciones en las MYPES se realiza en los procesos productivos, luego en nuevos productos y en los modelos de gestión (como cambios en las relaciones con proveedores, alianzas estratégicas, mejoras en canales de distribución, etcétera). Esto parece tener sentido en la medida en que la innovación en los pequeños negocios — tradicionalmente carentes de recursos-difícilmente se da en el desarrollo de nuevos productos, ya que ello requiere de inversión en investigación y desarrollo $(I+D)$, lo que para ellos no es económicamente viable. Sin embargo, en donde sí es posible innovar es en los otros ámbitos del negocio que por lo general van asociados a cambios de prácticas en las formas de hacer o interactuar. Esta información se puede ver en el gráfico 2.

\section{Gráfico 2: Tipos de innovación en pequeños negocio en Centroamérica (\%)}

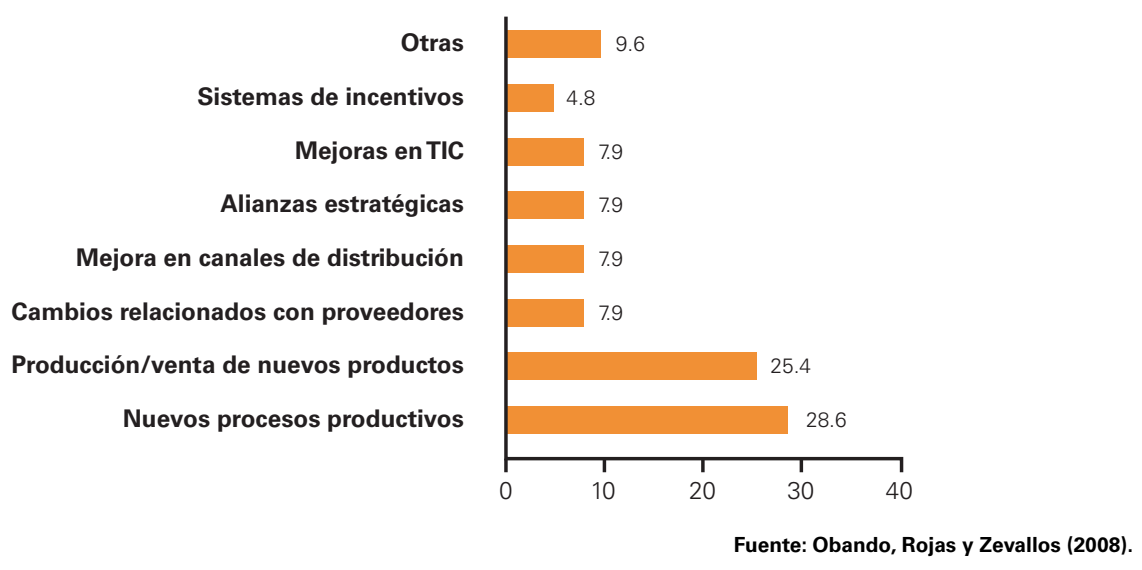

De otro lado, tenemos el tema de las competencias, que tradicionalmente se han centrado en el desarrollo de las llamadas "competencias laborales»; es decir, las que buscan que los trabajadores puedan desarrollar con suficiencia y eficiencia las actividades para las que están contratados. En este documento, nos orientamos más bien a las competencias con las que debe contar el empresario para liderar su negocio, conduciéndolo hacia el desarrollo competitivo y sostenible a mediano y largo plazo. Para ello, debe existir un cambio de enfoque que parte de cuestionar la premisa de base implícita: que el empresario

4. Una innovación tecnológica comprende la implementación de productos o procesos tecnológicamente nuevos o mejorados de forma significativa, mientras que la innovación no tecnológica comprende a la innovación de comercialización y a la innovación de la organización y gestión de la empresa. 
sabe lo que está haciendo, hacia dónde se dirige, a quién contrata, qué es lo que requiere, etcétera, y más bien se debe promover el desarrollo de competencias de los pequeños empresarios a fin de ayudarles en su desarrollo gerencial.

\subsection{Innovación: acción permanente en la empresa}

Sin intentar siquiera cuestionar la buena intención empresarial, la evidencia internacional muestra que la mayor parte de los pequeños negocios tiende a desaparecer antes de los dos años de fundados. Las razones son variadas, pero finalmente se llega a una conclusión: hay un mal cálculo (exceso de optimismo) de costos y/o beneficios, asociado a expectativas muy altas sobre los beneficios esperados del negocio.

Es por ello que, dado que en el proceso de aprendizaje del negocio el empresario juega un papel central, el desarrollar innovación en una empresa es una labor que debe ser liderada (mas no siempre ejecutada) por el empresario. Ella es por lo general resultado de la búsqueda de soluciones para resolver un problema o aprovechar una oportunidad de negocios. De esta forma, una vez definido el problema, la buena práctica señala la necesidad de buscar información sobre el mismo (por lo general, soluciones dadas en otros contextos) y sobre ella se deberían gestionar y evaluar las ideas presentadas para luego establecer las posibles soluciones en función de criterios definidos de antemano (tales como costos, empleo, etcétera) y, finalmente, ejecutar la propuesta de negocio. Lamentablemente, lo señalado antes es más la excepción que la regla. Por lo general, al empresario «se le ocurre algo" para resolver el problema y lo implementa (con mayor o menor éxito).

Por ello, y más allá del proceso de innovación en sí mismo, existen condiciones iniciales en donde las competencias del empresario juegan un papel central. Si las tiene el negocio, saldrá adelante; si no, es necesario que las desarrolle lo más pronto posible. Entre ellas destacan:

- La "capacidad de absorción» (absorptive capacity ${ }^{5}$ ), entendida como la habilidad que tienen las empresas (o más bien los empresarios) para reconocer el valor del nuevo conocimiento externo (así como interno), asimilarlo y aplicarlo con fines comerciales (o su aplicación en los negocios como parte de la creación de valor) (Cohen \& Levinthal, 1990; Fogg, 2012).

- La "orientación al aprendizaje», entendida como esa voluntad del empresario por aprender permanentemente basada en el mundo del trabajo más que en otro ámbito (pero sin excluirlos). Así, el aprendizaje basado en problemas (problem-based learning o PBL), entendido como el proceso en el que los empresarios aprenden en un contexto de problemas reales y variados con los que se enfrentan en su día a día, es también un ámbito donde ellos desarrollan un conocimiento flexible, así como habilidades específicas para la resolución de problemas, y de forma autodirigida.

5. Para nuestros fines, es necesario indagar más respecto de si esta "capacidad de absorción» es una cualidad de los empresarios que van fortaleciendo con el tiempo, es algo que traen al proceso emprendedor o si más bien es algo que se construye a lo largo de la vida (desde cero). 
- La «disciplina», entendida como la facultad de los empresarios para establecer acciones y prácticas que llevan a cabo de forma sistemática y que son transversales al negocio, muy probablemente asociadas a su vida personal.

- La «tolerancia al fracaso», entendida como el elemento que permite al empresario aprender de los errores y re-emprender una tarea utilizando el conocimiento anterior como herramienta.

Adicionalmente, el propio empresario y su equipo de trabajo deben construir un entorno favorable al crecimiento del negocio (con sus clientes, proveedores, distribuidores, etcétera), de forma tal que ellos se conviertan también en una fuente de información y contactos para el empresario, y eso que se observa hacia afuera de la empresa debe estar presente también al interior de la misma, con el cliente interno (el colaborador). Un entorno laboral apropiado también es fuente de innovación centrada en el equipo de trabajo. A continuación, se muestra la síntesis del proceso innovador en el gráfico 3.

\section{Gráfico 3: El proceso innovador}

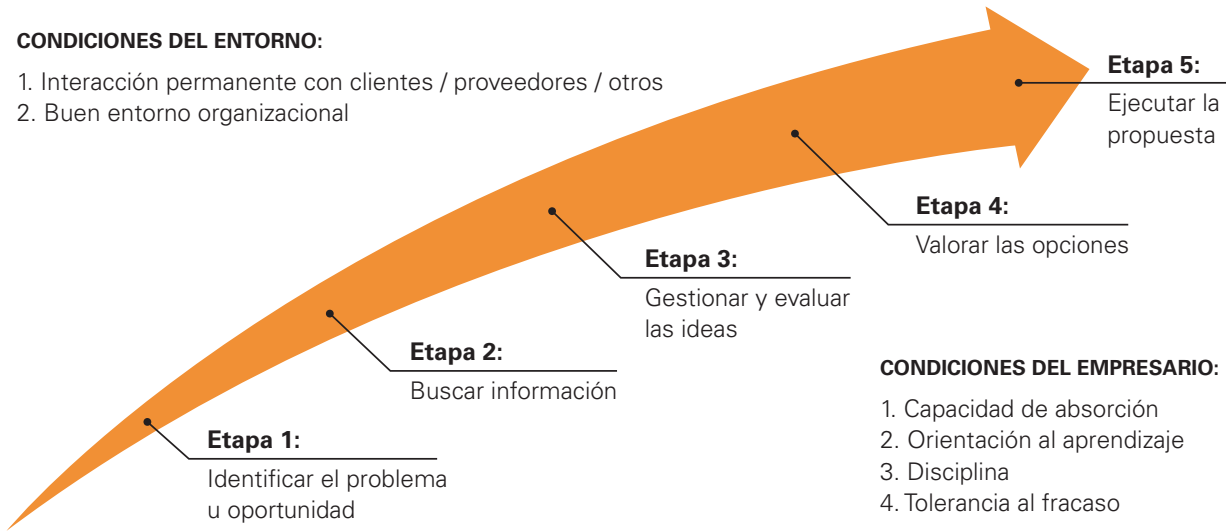

Fuente: elaboración propia sobre la base de Dornberger, Suvelza \& Bernal (2011).

Asimismo, la nueva economía, en la cual las tecnologías de la información y comunicación (TIC) se hacen protagonistas del cambio y la innovación supone para el empresario nuevos y más complejos retos. De hecho, por medio de las TIC se desarrollan nuevos modelos de negocios en donde son las pequeñas unidades económicas las que juegan un papel cada vez más importante. Ejemplos de ello son la empresa de transporte Uber, el sistema de alquiler de alojamientos Airbnb e incluso la app para aprender idiomas Duolingo, que nacieron en pequeña escala pero se han desarrollado rápidamente gracias al uso de las TIC y alcanzan en la actualidad el ámbito internacional.

Esto plantea de inicio la redefinición de los enfoques orientados al desarrollo emprendedor en las MYPES. Por un lado, el apoyo para desarrollar y consolidar empresas que tienen opción de ser sostenibles y, del otro, la asistencia a estas unidades económicas de subsistencia para tratar de equiparar oportunidades, llevándolas en lo posible a un umbral de crecimiento.

En este enfoque se encuentra el gran desafío de distinguir a las iniciativas empresariales que tienen opción de desarrollo de las que están más cercanas a la subsistencia. Ambos 
grupos deben ser sujetos de acciones y políticas públicas, pero probablemente el primero tiene mayores posibilidades de escalar hacia nuevos y más significativos niveles de competitividad respecto del segundo. En esa línea, debe estar claro que no todas las empresas que nacen con tamaño pequeño lograrán ser empresas consolidadas en el mercado. Sin embargo, todas deberían contar con las mismas oportunidades de desarrollo y un entorno favorable -o al menos neutral - para su crecimiento, y deben ser sus talentos y competencias quienes definan en mucho su destino. De ahí la necesidad de plantear, a través de políticas públicas y/o privadas, acciones de apoyo y fomento al crecimiento de este tipo de agentes económicos.

\section{Metodología}

Nuestra hipótesis de investigación es que innovar en la MYPE no es imposible; por el contrario, es necesario y ya varios de estos agentes económicos lo están realizando para lograr un impulso de crecimiento y sostenibilidad, especialmente en sectores más tradicionales, donde la factibilidad de la innovación no se encuentra atada a recursos económicos tanto como a las propias habilidades de los emprendedores.

Para evidenciar esta hipótesis, en este trabajo se emplean y analizan los principales resultados de estadísticas secundarias tanto de Costa Rica como de Perú. Su finalidad es caracterizar a las MYPES, a sus conductores y al entorno de innovación en el que se desenvuelven a fin de hacer visibles sus competencias y el tipo de innovación en la que se centran. Sobre lo anterior, se considera que el tipo de innovación más frecuente es la no tecnológica, relacionada a la organización de sus procesos y modelos de gestión, frente a la innovación tecnológica, relacionada a nuevos productos.

Asimismo, se presentan un par de casos exitosos en generación de innovación incremental con el propósito de presentar medidas novedosas (innovación en el modelo de negocio) gracias a las cuales las MYPES fueron capaces de cambiar el rumbo cuando fue necesario.

\subsection{Análisis de estadísticas en Costa Rica y Perú}

En el caso de Costa Rica, "El estado de situación de las PYMES en Costa Rica 2013» (Ministerio de Economía, Industria y Comercio [MEIC], 2014) revela una realidad no muy diferente del resto de los países de la región; sin embargo, cabe señalar que más que PYME o MYPE, el término más utilizado es el de MIPYME (micro, pequeña y mediana empresa), las cuales representan el 95\% del universo empresarial. Del conjunto de las MIPYMES, el $74 \%$ es micro, el $12 \%$ es pequeña y casi el $14 \%$ es mediana empresa. El $42 \%$ de ellas se encuentra en el sector servicios; el $41 \%$, en el comercio; el $11 \%$, en la industria; y luego otros sectores de menor importancia. En cuanto al empleo, el 27\% del mismo lo genera la MIPYME.

Respecto a su participación en el comercio exterior, si bien la estadística refleja más de 2000 empresas exportadoras PYMES ${ }^{6}$, su aporte al valor exportado sigue siendo

6. Cabe señalar que PROCOMER, la oficina de comercio exterior de Costa Rica, define PYME como unidad económica con menos de 100 trabajadores, por lo que incluye a la micro en esta clasificación, pero sin desagregarla, difiriendo de la definición oficial, que es una fórmula que pondera activos, empleo y ventas. 
minúsculo respecto de la gran empresa. De acuerdo a cifras del Banco Central de Costa Rica, la aportación calculada de las PYMES al PBI (producto bruto interno) es de 30\%. En cuanto a su distribución espacial, el 77\% de las pymes se ubica en la región central (que comprende la capital San José y las áreas periféricas denominadas "Gran Área Metropolitana» o GAM).

En el caso peruano, las estadísticas más recientes del Ministerio de la Producción (Produce), tomadas del informe Las MIPYMES en cifras 2013, igualmente se refiere a MIPYMES ${ }^{7}$ y señala que, en el año 2013 , el $94.6 \%$ de las empresas formales eran microempresas y el $4.6 \%$ eran pequeñas empresas, como se observa en el tabla 1.

Tabla 1: Empresas formales en Perú (2013). Según estrato empresarial*

\begin{tabular}{l|c|c} 
estrato empresarial & número de empresas & porcentaje \\
\hline Microempresas & 1439778 & $94.6 \%$ \\
Pequeña empresa & 70708 & $4.6 \%$ \\
Mediana empresa & 2520 & $0.2 \%$ \\
\hline Total MIPYMES & 1513006 & \\
Gran empresa & 8306 & $0.5 \%$ \\
\hline Total de empresas & $\mathbf{1 5 2 1 3 1 2}$ & $\mathbf{1 0 0 . 0 \%}$
\end{tabular}

*El estrato empresarial es determinado según la Ley 30056. Se considera gran empresa a aquella cuyas ventas anuales son mayores a 2300 UIT.

Fuente: Ministerio de la Producción, 2014. Las MIPYMES en cifras 2013.

De acuerdo a las estadísticas del Ministerio de la Producción (Produce, 2014), en 2013 más de la mitad del número de MIPYMES tuvo ventas anuales menores o iguales a 13 UIT (S/ 48 000), los sectores comercio y servicios fueron los preferidos por las MIPYMES (aproximadamente $85 \%$ del total) y, a nivel geográfico, es en la capital del Perú y en la Provincia Constitucional del Callao donde más MIPYMES se han establecido $(48 \%$ de las formales). Asimismo, la contribución de las microempresas formales (menos de 10 trabajadores) al empleo peruano, de acuerdo a esta fuente, fue $70.5 \%$ del empleo en el sector privado en 2013.

En cuanto a su participación en las exportaciones, como en el caso de Costa Rica, la estadística de Produce en Perú refleja una participación significativa de 5845 MIPYMES exportadoras (75\% de las empresas exportadoras), pero su aporte al valor exportado sigue siendo pequeño (menos del 5\%) respecto de la gran empresa.

Todo lo anterior muestra una situación semejante a la encontrada en la mayor parte de países latinoamericanos, quizás con un par de diferencias importantes: el grado de formalización y el uso de la tecnología.

7. El criterio para definir el estrato empresarial en el Perú es el de la Ley 30056 (de 2013), según la cual si una empresa tiene un promedio de ventas anuales por debajo de las 150 UIT es microempresa, si fluctúa entre 150 y 1700 UIT es pequeña empresa y si está entre 1700 y 2300 UIT es una mediana empresa. 
En Costa Rica, y para las PYMES registradas ante el MEIC (registro PYME), cerca del $100 \%$ de ellas se encuentran registradas en tributación y por encima del $70 \%$ bien sea en la seguridad social o con un seguro de riesgos del trabajo ${ }^{8}$. En el caso del uso de la tecnología, para 2011 las microempresas registradas contaban en un 58\% con equipo de cómputo; las pequeñas, en un $87 \%$; y las medianas, en un $97 \%$. De ellas, tenían acceso a Internet el $56 \%$ de las microempresas, el $86 \%$ de las pequeñas y el $96 \%$ de las medianas. Lo anterior refleja una importante cobertura en las empresas de menor tamaño y, al mismo tiempo, una adecuada posición inicial para el acceso a información respecto de innovaciones. Cabe señalar que la cobertura de Internet en Costa Rica es una de las más altas de América Latina.

Para el caso de Perú, de acuerdo a la estimación realizada en el informe del Ministerio de la Producción (2014, p. 76), el número de MYPES (micro y pequeñas empresas) informales en 2013 fue ligeramente superior al número de MYPES formales (registradas en el padrón de contribuyentes de la Superintendencia de Administración Tributaria - SUNAT), las cuales ascienden a poco más de 1500 000). En relación al uso de TIC, de acuerdo a los Resultados de la encuesta de micro y pequeña empresa 2013 (INEI, 2014), que analiza información de 2012 de empresas con ventas netas entre 20 y 1700 unidades impositivas tributarias (UIT de 2012: S/ 3650) ${ }^{9}$, "el $67 \%$ de las micro y pequeña empresas manifestaron contar por lo menos con una computadora de escritorio, el $48.8 \%$ un equipo multifuncional, el $35.1 \%$ una computadora portátil, el $27.3 \%$ una impresora, el 23.5\% un teléfono con acceso a Internet (smartphone), el $8.6 \%$ un escáner y el $6.0 \%$ una fotocopiadora» (INEl, 2014, p. 51). Asimismo, "el 90.9\% de las micro y pequeñas empresas que tenían computadora de escritorio o laptop, declararon contar con servicios de Internet, el $0.9 \%$ declaró tener intranet y el $9.0 \%$ declaró no contar con servicios informáticos» (INEI, 2014, p. 53).

De otro lado, de acuerdo a los indicadores nacionales de ciencia, tecnología e innovación de Costa Rica en 2013 (que miden en lo fundamental los esfuerzos de las empresas en los sectores manufactureros, de energía y telecomunicaciones en la pequeña, mediana y gran empresa, sin considerar la micro), un 93.2\% de las empresas de estos sectores han realizado al menos algún tipo de actividad de innovación en los últimos años. Asimismo, un $70 \%$ de ellas han invertido en actividades de I\&D interna ${ }^{10}$ y un $66.6 \%$ lo hacen en procesos de capacitación para fomentar la innovación. En ese sentido, las actividades de innovación en procesos y productos han sido de gran relevancia,

8. Cabe señalar que el registro PYME del MEIC no es obligatorio, pero se requiere si la empresa desea recibir asistencia técnica o algún beneficio fiscal. Para obtenerlo y ser considerada PYME, la empresa debe cumplir con dos de los tres criterios de formalización: estar registrada en el Ministerio de Hacienda, en la Seguridad Social o bien contar con una póliza de riesgos del trabajo.

9. Para esta encuesta, el INEI consideró como microempresa aquella que tiene de uno hasta 10 trabajadores y un nivel de ventas anuales hasta un monto máximo de 150 UIT (S/ 547500 ) y como pequeña empresa aquella que tiene de uno hasta cien trabajadores y un nivel de ventas anuales entre 150 y 1700 UIT (S/ 6205 000).

10. La I\&D interna puede ser orgánica (que se da de manera espontánea y sin una estrategia concreta para generarla) o sistémica (que está respaldada por una práctica definida por la organización). La innovación orgánica es generada por algún integrante de la organización. La innovación externa se puede dar por adquisición, colaboración con otras organizaciones o por una política de desarrollo de proveedores innovadores. En general, la innovación ya no parte de la premisa de que las nuevas ideas deben producirse al interior de las empresas, sino que deben ser el resultado de personas creativas (muchas veces externas) que no son parte de la empresa. 
pero también se consideran significativas la innovación en organización y comercialización, como se aprecia en el tabla 2.

Tabla 2: Porcentaje de empresas que han realizado actividades orientadas a la innovación (Costa Rica, 2012-2013) Actividades orientadas a cambios, mejoras y/o innovaciones en...

\begin{tabular}{|c|c|c|c|c|c|}
\hline & producto & proceso & organización & comercialización & $\begin{array}{l}\text { cualquier tipo de } \\
\text { innovación }\end{array}$ \\
\hline i+D interna & 58.1 & 43.9 & 20.3 & 25.9 & 70.0 \\
\hline I+D externa & 13.3 & 7.0 & 2.3 & 6.3 & 17.3 \\
\hline $\begin{array}{l}\text { Bienes de capital } \\
\text { (maquinaria y equipo) }\end{array}$ & 29.5 & 44.6 & 7.9 & 9.2 & 57.4 \\
\hline Hardware & 13.1 & 24.3 & 28.8 & 17.6 & 48.9 \\
\hline Software & 12.8 & 24.3 & 26.8 & 16.7 & 44.1 \\
\hline $\begin{array}{l}\text { Contratación de } \\
\text { tecnología (existente) }\end{array}$ & 17.3 & 18.2 & 9.9 & 6.8 & 29.1 \\
\hline $\begin{array}{l}\text { Ingeniería y diseño } \\
\text { (industrial) }\end{array}$ & 16.7 & 21.6 & 6.1 & 4.5 & 27.7 \\
\hline Gestión (in house) & 22.7 & 27.3 & 18.2 & 11.5 & 38.3 \\
\hline Capacitación & 32.9 & 46.6 & 35.4 & 22.7 & 66.6 \\
\hline $\begin{array}{l}\text { Consultorías (para } \\
\text { cambios novedosos) }\end{array}$ & 9.0 & 12.4 & 13.5 & 7.2 & 22.7 \\
\hline $\begin{array}{l}\text { Al menos algún tipo de } \\
\text { actividad de innovación }\end{array}$ & 77.9 & 81.5 & 65.1 & 52.9 & 93.2 \\
\hline
\end{tabular}

Nota: valores respecto al $93.5 \%$ de las empresas que realizaron actividades dirigidas a generar innovación en el período 2012-2013.

Fuente: MICIT (2015).

Lo anterior sustenta la tesis del tipo de innovación más frecuente y, por tanto, la necesidad de acciones de fomento en ese tipo de iniciativas. Este mismo fenómeno se observa por tamaño de empresa, donde la inversión en innovación se encuentra altamente diversificada en un conjunto de acciones que buscan no solo mejorar las condiciones objetivas del negocio (bienes de capital), sino también mejorar las competencias de los involucrados en el proceso por medio de acciones orientadas a la gestión o bien la capacitación, entre otras. Ello se aprecia en el gráfico 4. 


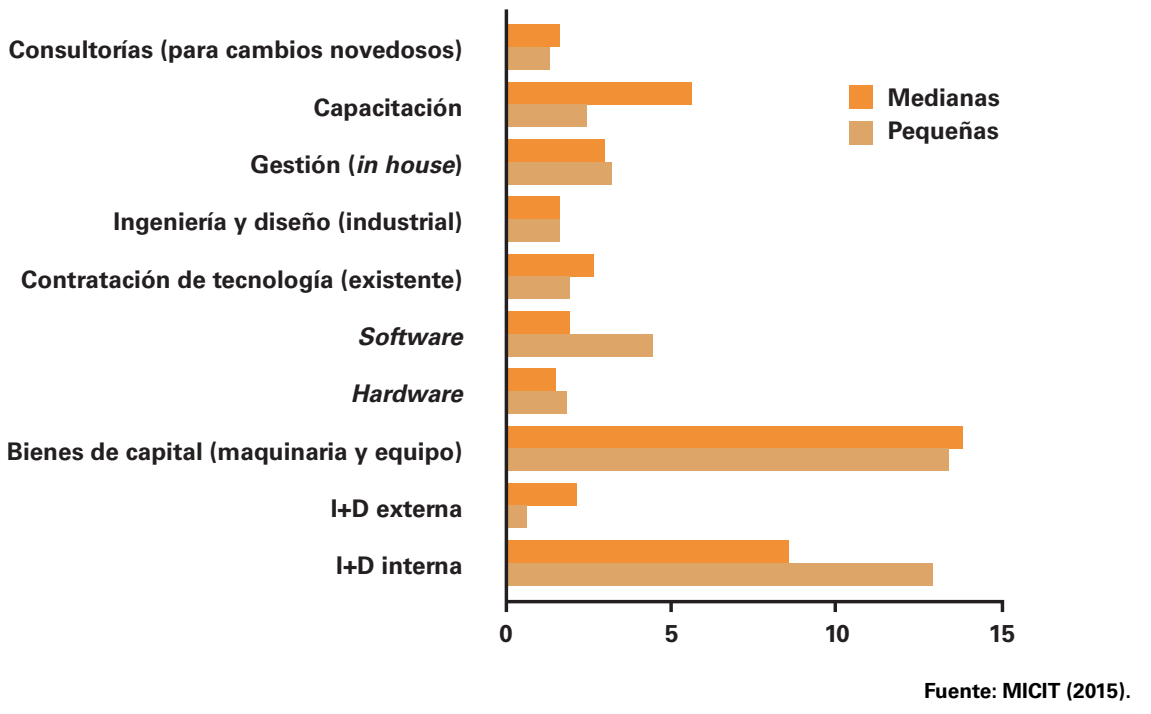

Como observamos en el gráfico 2, el tipo de innovación de la subregión se centra más en procesos y modelos de gestión que en productos. En el gráfico 4, ello vuelve a aparecer, esta vez con una tendencia —-más centrada en el caso de las pequeñas empresashacia la innovación interna antes que a la externa (que sí es el caso de las medianas), lo cual podría estar asociado al nivel de madurez del negocio. Es decir, conforme el negocio se consolida, busca conocer más las características del cliente (gustos y preferencias), mientras que, cuando el negocio es pequeño, la apuesta se centra en el producto y su valor agregado. Algo parecido ocurre con la capacitación pues las empresas, conforme van creciendo, identifican oportunidades de mejora en la mayor calificación de su personal.

Igualmente, en el caso peruano, a partir de los Resultados de la encuesta a micro y pequeña empresa 2013 (INEI, 2014), se evidencian los siguientes comportamientos a partir de las características encontradas en el análisis de los resultados:

- $\quad$ Conductores de MYPES flexibles y menos adversos al riesgo: dado que el $55.8 \%$ de los propietarios manifestó tener entre 25 y 49 años de edad, y que el $33.4 \%$ de los propietarios tiene estudios de secundaria completa y el $23 \%$ educación universitaria completa, consideramos que se podrían redefinir más fácilmente estrategias organizacionales en sus empresas.

- Conductores de MYPES trabajan de manera aislada: solo el 5.4\%, de estas unidades económicas formaba parte de alguna organización con fines empresariales, de las cuales el $72.5 \%$ declaró que el tipo de organización al que pertenecían era la asociación. El 69.1\% de las MYPES que pertenecían a alguna organización con fines empresariales manifestó que lo hicieron para acceder a mercados; el 41.8\%, para acceder a información y asistencia técnica; y el $38.4 \%$, para negociar con proveedores (ver tabla 3). Asimismo, el 62\% de las MYPES no asociadas a alguna organización con fines empresariales señaló que el principal motivo para ello era la falta de conocimiento. Esto 
muestra que habría un potencial para promover la formación de asociaciones y obtener los resultados favorables que ello promueve.

Tabla 3: Motivos de las MYPES para pertenecer a una organización con fines empresariales en Perú (2012, porcentajes de participación)

\begin{tabular}{l|c} 
MYPES & $\mathbf{6 4 9}$ \\
\hline Para negociar con proveedores & 38.4 \\
Para acceder a servicios financieros & 13.8 \\
Para acceder a mercados & 69.1 \\
Para acceder a información empresarial & 30.2 \\
Para acceder a información y asistencia técnica & 41.8 \\
Otro* & 3.3 \\
Nota: el resultado corresponde a una pregunta con respuesta múltiple. \\
* Considera, como otros motivos, los beneficios laborales y local para negocio.
\end{tabular}

Fuente: INEI (2014).

- Hay aún poco interés de parte de los conductores de las MYPES en lo que a capacitación de gestión empresarial moderna"11 se refiere —solo el 19\% había participado en eventos de capacitación relacionados con la gestión empresarial—, prefieren capacitarse en marketing (19\%), calidad (10.2\%), exportaciones $(10.1 \%)$ y formalización $(9.7 \%)$. Es de destacar que la mayor asistencia se ha dado al interior del país: en Ayacucho (50\%), Huancayo (37.9\%), Piura (27.4\%) y Cusco $(26.8 \%)$.

- Los cursos menos seguidos fueron negocios por Internet (2.1\%), marcas y franquicias $(2.0 \%)$ y cadena de suministros $(1.2 \%)$. Se debe comentar también que, de acuerdo a la encuesta, solo el $6.5 \%$ de los conductores de las MYPES declararon que habían participado en eventos de servicios sobre nuevas tecnologías para la gestión de su empresa.

- $\quad$ Sobre cursos y/o de servicios TIC, el 7.8\% de los conductores de las MYPES declaró que había participado en esos cursos, especialmente los aplicados a mercadotecnia, entre los cuales destacan: diseño de página web (21.6\%), operaciones de banca electrónica (18.2\%), transacciones comerciales con compradores por Internet $(16.0 \%)$ y transacciones comerciales con proveedores por Internet (14.4\%).

- Las principales capacitadoras de las MYPES son las empresas privadas e instituciones financieras: el $29.1 \%$ de los conductores de las MYPES que asistieron a eventos de gestión empresarial fueron capacitados por

11. De acuerdo a la encuesta Resultados de la encuesta de micro y pequeña empresa 2013 (INEI, 2014), los conocimientos aprendidos en gestión empresarial son aquellos que se emplean en la dirección, organización, planificación y control de las diferentes funciones de una empresa. 
empresas privadas e instituciones financieras, mientras que el $20.4 \%$ fue por el Gobierno (central, regional o local) y solo el $14.3 \%$ por los institutos de educación superior. Sin embargo, son de destacar las capacitaciones realizadas por el Gobierno en Ayacucho, Huancayo, Juliaca e Iquitos; por el sector privado en lquitos, Juliaca y Cusco; por las cámaras de comercio y colegios profesionales en Piura, Trujillo y Chiclayo; y por los institutos de educación superior en Cusco y Chiclayo, como se observa en la tabla 4.

- De la totalidad de los conductores de las MYPES que habían seguido una capacitación sobre gestión empresarial, el 93\% manifestó haber aplicado los conocimientos adquiridos en su empresa, siendo mercadeo y ventas (36.1\%), producción $(23.3 \%)$, dirección y gerencia (18.3\%), administración (15.6\%) y contabilidad y finanzas (5.9\%) las áreas de la empresa en las que se han aplicado los conocimientos aprendidos.

Tabla 4: Instituciones que capacitaron en cursos o eventos en gestión empresarial en Perú, según ciudad (2012)

\begin{tabular}{|c|c|c|c|c|c|c|c|c|c|}
\hline Ciudad & $\begin{array}{l}\bar{\pi} \\
\stackrel{\pi}{\circ}\end{array}$ & 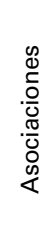 & 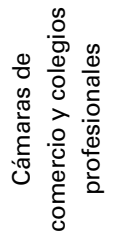 & 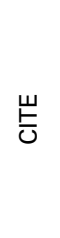 & 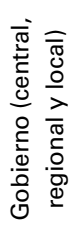 & 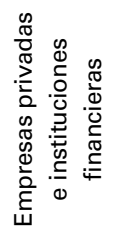 & 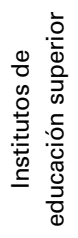 & $\begin{array}{l}\frac{0}{0} \\
\frac{0}{0} \\
\frac{\pi}{0} \\
\frac{0}{0} \\
\frac{0}{0} \\
\frac{1}{5} \\
5\end{array}$ & $\begin{array}{l}\infty \\
\stackrel{0}{0} \\
0\end{array}$ \\
\hline TOTAL & 100.0 & 6.9 & 16.4 & 0.7 & 20.4 & 29.1 & 14.3 & 11.5 & 0.6 \\
\hline Lima-Callao & 100.0 & 7.9 & 16.0 & 0.8 & 16.8 & 30.8 & 14.2 & 13.1 & 0.5 \\
\hline Arequipa & 100.0 & 3.7 & 7.1 & 0.0 & 28.4 & 29.4 & 18.6 & 10.8 & 2.1 \\
\hline Ayacucho & 100.0 & 0.0 & 7.4 & 0.0 & 74.1 & 3.7 & 7.4 & 7.4 & 0.0 \\
\hline Chiclayo & 100.0 & 0.0 & 22.6 & 0.0 & 32.3 & 9.4 & 35.8 & 0.0 & 0.0 \\
\hline Cusco & 100.0 & 8.8 & 0.0 & 0.0 & 17.6 & 35.3 & 38.2 & 0.0 & 0.0 \\
\hline Huancayo & 100.0 & 1.7 & 1.6 & 0.0 & 50.2 & 17.1 & 3.1 & 6.5 & 2.8 \\
\hline Iquitos & 100.0 & 5.0 & 0.0 & 0.0 & 40.0 & 39.9 & 15.1 & 0.0 & 0.0 \\
\hline Juliaca & 100.0 & 0.0 & 0.0 & 0.0 & 42.9 & 35.7 & 14.3 & 7.1 & 0.0 \\
\hline Piura & 100.0 & 2.9 & 37.9 & 0.0 & 17.5 & 17.5 & 11.7 & 12.6 & 0.0 \\
\hline Trujillo & 100.0 & 3.2 & 31.7 & 1.1 & 31.0 & 18.9 & 10.8 & 2.9 & 0.4 \\
\hline
\end{tabular}

Fuente: INEI (2014).

En la Encuesta nacional de innovación en la industria manufacturera 2012 (INEI, $2013)^{12}$ destaca que el $63 \%$ sean empresas innovadoras en la industria manufacturera. El

12. Esta encuesta fue aplicada a una muestra de 1220 empresas que realizaron actividades manufactureras y que obtuvieron ventas anuales mayores a 150 UIT. 
$55.8 \%$ de las empresas industriales han realizado alguna innovación tecnológica, sea en producto o en el proceso productivo; mientras que el 56.6\% desarrollaron innovación no tecnológica, es decir que innovaron en su organización empresarial o en comercialización. Como se aprecia en la tabla 5, las actividades de innovación en procesos y productos han sido importantes, pero tan significativas como ellas han sido las innovaciones en organización y comercialización.

Tabla 5: Conducta innovadora de las empresas de la industria manufacturera en Perú (2012)

\begin{tabular}{l|c|c} 
conducta innovadora & $\begin{array}{l}\text { porcentaje respecto al } \\
\text { total de empresas }\end{array}$ & $\begin{array}{l}\text { porcentaje respecto a las } \\
\text { empresas que realizaron A * }\end{array}$ \\
\hline Total de empresas & 100.0 & 100.0 \\
\hline Empresas que realizaron actividad de innovación & 65.5 & 96.7 \\
\hline Empresas innovadoras & 63.3 & 85.2 \\
$\quad$ Innovación tecnológica & 55.8 & 62.9 \\
$\quad$ Innovación en producto & 42.2 & 69.5 \\
Innovación en proceso & 45.5 & 86.4 \\
$\quad$ Innovión no tecnológica & 56.6 & 71.6 \\
$\quad$ Innovación en organización & 46.9 & 65.3 \\
\hline Empresas no innovadoras & 42.7 & 3.3 \\
\hline Empresas que no realizaron actividad de innovación & 2.2 &
\end{tabular}

Fuente: INEI (2013).

Asimismo, el gráfico 5 permite apreciar que, si se diferencia a las empresas por tamaño, la innovación no tecnológica en comercialización (cambios en diseño o empaque, nuevos métodos y técnicas de promoción, y colocación del producto y nuevos métodos de establecimientos de precios) ha sido más representativa en el caso de las pequeñas empresas, probablemente debido "al interés de atender a un consumidor cada vez más exigente e informado» (Quiroz, 2014, p. 22).

\section{Gráfico 5: Empresas por tamaño de la industria manufacturera con innovación no tecnológica en} comercialización en Perú

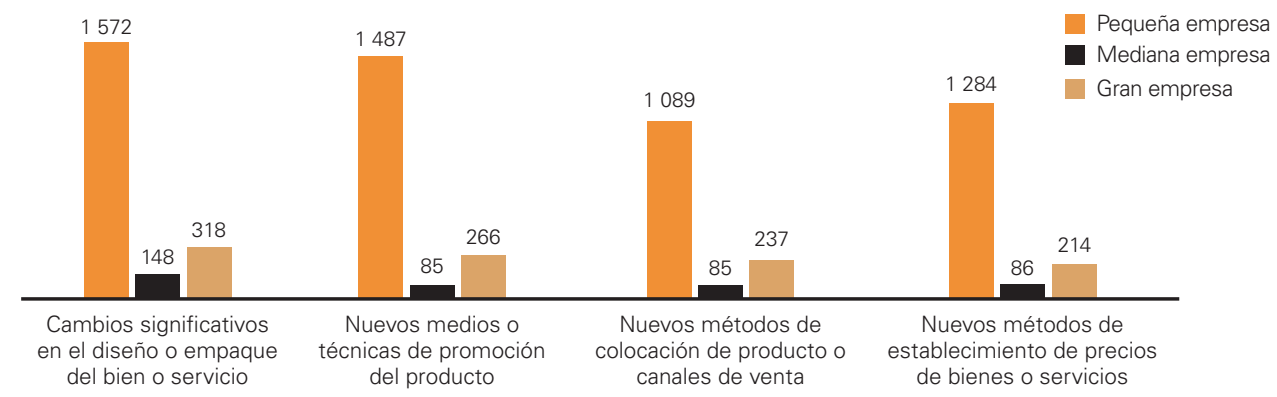

Fuente: Quiroz (2014, p. 23). 
La información comentada anteriormente permite señalar que, tanto en el caso de Costa Rica como en el de Perú, las actividades de innovación se encuentran asociadas más a procesos y a modelos de gestión que a la tecnología (en productos, esencialmente). En ese sentido, la política de capacitación de las MIPYMES que se observa en las estadísticas es parte de un proceso continuo de formación de capital humano, componente principal de una política empresarial de apropiación, adopción y adaptación tecnológica (Dini, Rovira \& Stumpo, 2014). Sin embargo, incluso los propios empresarios de MIPYMES no son conscientes de que varias de las actividades que están llevando a cabo son innovaciones: "El dueño de una pequeña empresa rechazó la idea de que alguna cosa que haya hecho su negocio podría ser clasificada como innovadora, diciendo No estamos creando el lpod» (Forbes, 2016).

Consideramos que lo que se requiere trabajar con las MYPES es un conjunto de medidas que las empoderen en su accionar para la toma de decisiones en un contexto digital y dinámico, lo cual pasa por definir una dirección clara en la estrategia de su negocio, priorizar una buena relación con sus clientes, mejorar sus competencias digitales, definir métricas claras que ayuden a conocer mejor a sus clientes y al mercado, y fortalecer su capacidad para cambiar el rumbo de su negocio cuando ello sea necesario. Esas fortalezas son las que tienen los dueños o gerentes de las empresas de los casos que presentamos en el siguiente acápite. No obstante, es importante rescatar que el desempeño exitoso de las empresas es resultado de múltiples factores combinados que se refuerzan mutuamente (Grazzi \& Pietrobelli, 2016, citado en Navarro \& Olivari, 2016, p. 41) y que la innovación es un componente. En esa línea, las prácticas (o competencias) empresariales determinan en muchos casos una senda exitosa de las empresas. Un empresario que aprende de forma permanente, responsable socialmente, que trabaja en redes y que desarrolla una gestión orientada a resultados, tenderá sin duda a generar innovaciones en su negocio o bien a promoverla en su equipo de trabajo (Zevallos, 2012).

\subsection{Análisis de casos}

Lo mencionado anteriormente — que hay MYPES realizando especialmente innovación incremental y no tecnológica- se hace visible en los casos a reseñar ${ }^{13}$ :

1. La cafetería Café de Mar se ubica en una zona comercial de alta concurrencia del centro de la ciudad. Su característica innovadora se hace visible desde que uno ingresa al local. No hay cocina o un área donde uno pensaría que se preparan alimentos, solamente una barra con una máquina para hacer café y unos refrigeradores con vitrina exhibidora. Dado lo anterior, se podría pensar que se preparan los productos en otro lugar y se traen a la cafetería. Así es, pero la diferencia es que no es el dueño quien trae los alimentos, sino pequeños productores de sándwiches, pasteles, empanadas, wraps y demás productos de su variada oferta.

Su negocio se ha convertido en la vitrina de un conjunto de microempresarios que tienen en Café de Mar la ubicación ideal para que los

13. Por razones de confidencialidad, se han modificado los nombres de las empresas y propietarios. 
clientes prueben sus productos. Hay por supuesto un modelo de negocio en el que el dueño cobra una pequeña comisión por producto vendido y al final del día hay un análisis conjunto de lo que tiene mayor demanda. En estos espacios de trabajo se reflexiona respecto de los comentarios de los clientes que son la retroalimentación para todos e insumos para mejorar la propuesta.

El dueño conoce a todos los proveedores y mantiene una buena relación con ellos. Quienes han mantenido la relación, en la mayoría de los casos, ya cuentan con una marca propia, han pasado por un curso de manipulación de alimentos que los certifica para la actividad y facturan formalmente con la empresa.

De esta forma, existe un proceso de selección de la mejor oferta para el local y los productores se afanan por lograr una oferta de calidad en precio y presentación. Si se quiere, se puede decir que es un modelo colaborativo tipo Uber o Airbnb, pero en el mundo real. El dueño de Café de Mar es el equivalente de la plataforma tecnológica pues ya no es él quien asume el riesgo, sino que lo diluye entre todos sus proveedores, les genera visibilidad y gana por ello. Es una PYME que trabaja conjuntamente con otras de forma colaborativa. ¿Se puede decir que es un modelo de negocios innovador? Sin duda. Ha resuelto uno de los problemas más importantes de las PYMES al ingresar el mercado: las malas estimaciones, pues el dueño ajusta todos los días las mismas y de esa forma minimiza el riesgo de lanzar un producto sin demanda.

2. El otro caso es aún más sencillo en términos de prácticas, pero de gran valor innovador. Doña Meche es una experta en la cocina y sus habilidades han sido reconocidas por los estudiantes de una conocida universidad capitalina frente a la que ella vive y trabaja. Inicialmente, el garaje de su casa se convirtió en un comedor; pero, conforme fue aumentando su fama, también tuvo que ceder espacios de su sala y comedor para ampliar su área de atención. Cuando llegaron estudiantes de Gestión Empresarial e Ingeniería Informática, vieron en doña Meche una oportunidad de negocios. Ellos necesitaban realizar una práctica y le propusieron a doña Meche crearle un sitio web para que pueda vender sus productos también por Internet (a la manera de un catering service).

La oportunidad que detectaron los estudiantes fue la de ofrecer el producto vía Internet a la gran cantidad de connacionales que vive fuera del país y a quienes les gustaría hacerle una atención a su familia en su cumpleaños, matrimonios, etcétera. De esta forma, no solo se creó el sitio web, sino también una propuesta publicitaria más allá de las fronteras que le permitió no solo expandir el negocio a nivel internacional, sino también bancarizar a doña Meche, quien hasta ese momento no tenía una cuenta en el sistema financiero, necesaria para el pago con tarjeta o PayPal.

Así las cosas, a los pocos meses del desarrollo del sitio web, doña Meche tuvo que cerrar la cafetería para los estudiantes de la universidad, 
ya que necesitaba el espacio para producir y embalar las cantidades de pedidos que le llegaban desde diferentes lugares (almuerzos, despedidas, matrimonios, cumpleaños, etcétera), para ser atendidos por ella, dada la calidad de sus productos y sus módicos precios.

De esta forma, un negocio convencional (de comidas), por medio de una innovación en su modelo de negocio, tuvo un impacto inusitado y mejoró considerablemente los ingresos de doña Meche.

\section{Resultados y hallazgos}

Uno de los puntos más importantes que pretendemos evidenciar en este documento es que la innovación no es monopolio de las grandes empresas o de las más dinámicas, sino que también existe en las MYPES y que no requiere de cuantiosas inversiones o de conocimientos técnicos avanzados, pero sí de una cultura orientada al aprendizaje y al cambio. Es posible que cada empresa, en función de sus conocimientos, sus competencias, su nivel de desarrollo y su madurez empresarial, genere innovaciones incrementales que mejoren su capacidad competitiva.

Los casos presentados muestran cómo un cambio en la forma de comercializar termina siendo no solo una innovación, sino que genera un espacio de trabajo para varias MYPES que pueden ahora incrementar el espectro de visibilidad de su oferta gastronómica. Lo mismo con una innovación tecnológica poco costosa, empleando TIC que modifican el modelo de negocios y atraen un mercado nuevo y poco explorado.

Sin embargo, para lograr innovaciones que contribuyan a la sostenibilidad de los negocios, es necesario desarrollar una «cultura de innovación» en las empresas, que supone un cambio en los paradigmas organizacionales y nuevas prácticas de interrelación entre ellas. En los casos antes señalados observamos, de un lado, que la interrelación permanente con los proveedores por medio de las conversaciones del final del día les aportan a ellos y al propio empresario un conocimiento y una retroalimentación que permiten ajustar la oferta en plazos muy cortos, con lo que se minimizan las pérdidas y se posibilita la mejora continua en el negocio, competencia central en un modelo de negocios exitoso. De otro lado, la apertura a la innovación y las nuevas ideas posicionó a una empresaria en un mercado que para ella era totalmente ajeno.

Podemos decir que la innovación en las MYPES es un proceso que surge en el emprendedor o su entorno, pero que este debe impulsarlo en su equipo para desarrollarlo; es decir, es un proceso permanente en la empresa y requiere visión, actitud, escucha, asumir riesgos, autocrítica y resiliencia.

\section{Implicancias y conclusiones}

La primera implicancia del presente artículo es visibilizar a la innovación como un evento accesible a cualquier tipo y tamaño de negocio. La evidencia presentada muestra que la innovación no requiere necesariamente de una inversión económica o conocimientos técnicos significativos tanto como de una propensión hacia el aprendizaje y una forma más colaborativa de trabajo con clientes y proveedores. El hecho de que los casos pertenezcan 
a un sector económico tradicional es adrede, pues nos interesa enfatizar la accesibilidad a la innovación por parte de los estratos empresariales más pequeños y tradicionales, aunque el reto a futuro es incorporar otros sectores —naturalmente poco dinámicos— e identificar las condiciones que les permitan mejorar sostenidamente su productividad.

Una segunda implicancia es que las MYPES, tanto en Costa Rica como en Perú, tienen conectividad (importante porcentaje de acceso a TIC: equipos de cómputo e Internet), pero les falta mayor capacitación en su uso con fines gerenciales y empresarial en gestión moderna, empleando Internet o servicios sobre TIC para la gestión de la empresa. Por ello, la política de capacitación a PYMES debería también orientarse a estos temas a fin de mejorar su competencia digital en negocios.

Otra de las implicancias tiene que ver con cómo la innovación puede acelerar la formalización de la empresa. Fuera de consideraciones normativas respecto de su importancia en los negocios, la formalización es resultado del proceso de desarrollo del empresario y aparece como un requerimiento de sus clientes y no como consecuencia de incentivos o castigos. El primer caso señalado es un buen ejemplo de cómo la sostenibilidad de la relación de negocios contribuyó decisivamente a encaminar a varios de los proveedores hacia la formalización.

Así, la lección es sobre la importancia de innovar para acelerar el crecimiento y, con ello, la formalización. Sobre el particular, en varios países de la región se han desarrollado actividades orientadas a la formalización bajo la premisa normativa (la necesidad de "formalizar» a los informales y que "contribuyan» fiscalmente); sin embargo, la efectividad de las mejoras se encuentra asociada a una "mezcla de políticas», como señala la OIT (2014) en su enfoque integrado. Además de mejorar la capacidad económica (productividad por medio de mejora en las competencias, gestión, etcétera), como en el caso señalado en este documento, la innovación plantea la necesidad de modificar las normas, mejorar los incentivos y la fiscalización. Por medio de esta "estrategia integrada" se han generado resultados importantes en varios países de la región. Este es un llamado de atención a los Gobiernos para que superen el enfoque parcial de la formalización como mecanismo para reducir la hipotética competencia desleal con las empresas formales, o para dejar de pensar que los incentivos per se son suficientes para formalizar los negocios. 


\section{bibliografía}

Amorós, J.; M. Guerra \& J.

\section{Carrillo}

Global Entrepreneurship Monitor. Reporte

Nacional de Chile 2007. Santiago de Chile:

Universidad Adolfo Ibáñez, Universidad del

Desarrollo.

\section{Berry, Albert}

2002

Valoración de políticas de apoyo a la

pequeña empresa: primera aproximación a

una metodología regional (Serie de Buenas

Prácticas del Departamento de Desarrollo

Sostenible). Washington D.C.: Banco

Interamericano de Desarrollo.

\section{Cohen, Wesley \& Daniel Levinthal}

1990

«Absorptive capacity: A new

perspective on learning and innovation".

Administrative Science Quarterly, 35 (1),

pp. 128-152. Disponible en: <https://doi.

org/10.2307/2393553>

\section{Congreso de la República del Perú}

2013

Ley No 30056. Ley que modifica diversas

leyes para facilitar la Inversión, impulsar

el Desarrollo Productivo y el Crecimiento

Empresarial. Disponible en: <http://www.

proinversion.gob.pe/RepositorioAPS/0/0/

arc/MOXI LEY 30056/ley30056.pdf>.

\section{Congreso Nacional de Chile}

2008

Ley N²0.267. Crea el Sistema Nacional de

Certificación de Competencias Laborales

y perfecciona el Estatuto de Capacitación

y Empleo. Disponible en: <http://www.

leychile.cl/Navegar?idNorma=272829>
Dini, Marco; Sebastián Rovira \& Giovanni Stumpo (comps.)

2014

Una promesa y un suspirar: políticas de innovación para PYMES en América Latina. Santiago de Chile: CEPAL, Cooperación Alemana.

\section{Dornberger, Utz; Alfredo Suvelza \& Luis Bernal (eds.)}

2011

Gestión de la fase temprana de la innovación. Leipzig: In4in, SEPT, Conoscope, GIZ, CENPROMYPE.

\section{Fogg, Helen}

«Tracing the links between absorptive capacity, university knowledge exchange and competitive advantage in SMEs". International Journal of Entrepreneurship and Innovation, 13 (1), pp. 35-44. Disponible en: <https://doi.org/10.5367/ijei.2012.0061> .

\section{Forbes}

2016

Lecciones de innovación de las pequeñas empresas. Disponible en: $<$ http://www.delfinoconegocios.com/ single-post/2016/10/10/Lecciones-deinnovaci\%C3\%B3n-de-las-empresaspeque $\%$ C3\%B1as $>$.

\section{INEI}

Perú: Encuesta nacional de innovación en la industria manufacturera, 2012.

Principales resultados. Disponible en: <http://www.inei.gob.pe/media/ MenuRecursivo/publicaciones digitales/ Est/Lib1076/index.html>.

\section{INEI}

\footnotetext{
Resultados de la encuesta de micro y pequeña empresa 2013. Disponible en: <http://www.inei.gob.pe/media/ MenuRecursivo/publicaciones digitales/ Est/Lib1139/index.html>.
} 
Kantis, Hugo; Juan Federico \& Sabrina Ibarra

Condiciones sistémicas para el

emprendimiento dinámico. Novedades

y tendencias para fortalecer e integrar

los ecosistemas de la región. Rafaela:

Asociación Civil Red Pymes Mercosur.

Lebendiker, Marcelo; Emilio

Zevallos, Eduardo Alonso \&

\section{Petra Petry}

2010

Diagnóstico sobre la situación del emprendedurismo en Centroamérica.

San Salvador: KFW, GTZ-Desca,

CENPROMYPE.

\section{MICIT}

Indicadores Nacionales 2013 Ciencia,

Tecnología e Innovación. Subsistema

Nacional de Indicadores de Ciencia,

Tecnología e Innovación. San José: MICIT.

Ministerio de Economía, Industria y Comercio (MEIC)

El estado de situación de las PYMES en

Costa Rica 2013. Disponible en: <http:// www.meic.go.cr/documentos/thm14p03s/ EstadoSituacionPYME2013.pdf>.

\section{Ministerio de la Producción}

2013

Encuesta nacional de innovación en la industria manufacturera 2012. Disponible en: < http://www.perucam.com/perucam new/pdf/ei/EncuestaNacional.pdf $>$.

\section{Ministerio de la Producción}

2014

Las MIPYMES en cifras 2013.

Disponible en: <http://demi.produce.

gob.pe/images/publicaciones/

publi7f308c9e5f8468f9c_63.pdf>.
Navarro, Juan Carlos \& Jocelyn Olivari (eds.)

La política de innovación en América Latina

y el Caribe. Nuevos caminos. Washington

D.C.: BID. Disponible en: <https://doi. org/10.18235/0000338>

\section{Obando, Juan Carlos; Liliana} Rojas \& Emilio Zevallos

Características de la microempresa y sus necesidades de capacitación en Centroamérica y República Dominicana. San José: Coordinación Educativa y Cultural (CECC), OIT, FOIL, OLACD.

The Organisation for Economic Co-operation and Development (OECD)

Globalisation and Small and Medium Enterprises (SMES). Volume I: Synthesis Report. París: OECD Publishing.

\section{Organización Internacional del Trabajo (OIT)}

Panorama Laboral Temático 1: transición a la formalidad en América Latina y el Caribe. Lima: Oficina Regional para América Latina y el Caribe.

\section{Quiroz, Consuelo}

2014

La sostenibilidad de la innovación en la industria manufacturera peruana. Lima: INEI.

\section{Villarán, Fernando \& Iván} Mifflin (comps.)

Creando riqueza desde abajo. Las micro y pequeñas empresas en el Perú. Lima: Fondo Editorial del Congreso. Disponible en: $<$ http://blog.pucp.edu.pe/blog/wp-content/ uploads/sites/479/2011/02/827.pdf>. 


\section{bibliografía}

\section{Zevallos, Emilio}

2001 Empresa Media, una nueva clasificación.

Comercio Exterior, 50 (3), pp. 230-235.

\section{Zevallos, Emilio}

2003 «Micro, pequeñas y medianas empresas en América Latina». Revista de la CEPAL, 79, pp. 53-70.

\section{Zevallos, Emilio}

2012
El modelo de desarrollo empresarial.
(Documento de trabajo 003). San José:
Fundación Omar Dengo. Disponible en:
$<$ http://www.fod.ac.cr/avanza/images/
documentos/Modelo-de-Desarrollo-
Empresarial.pdf > .

Fecha de recepción: 16/03/2016

Fecha de aceptación: 11/10/2016 